\title{
Diabetes is a disease characterized by impaired proteostasis
}

\begin{abstract}
The discovery of insulin production by the pancreatic islet cell that required removal of the exocrine pancreas was a milestone discovery in endocrine medicine. It later became known that insulin synthesis is disrupted with the production of amyloid from amyloid fibrils, and that this process led to the burnout of the islet cell. It also was learned that the development of renal insufficient and failure in diabetes mellitus is associated with hyaline granules in the glomerulus as well as micro vascular disease. This process of protein misfolding disorder in either case is fundamentally a diabetic proteomic feature, which is also expressed in the brain under a number of circumstances, some of which are genetic.
\end{abstract}

Keywords: insulin, pancreatic islet, diabetes, hyperglycemia, amyloid, proteostasis
Volume I Issue 3 - 2017

\author{
Larry H Bernstein \\ Wayne State University School of Medicine, USA
}

Correspondence: Larry H Bernstein, Wayne State University School of Medicine, FCAP54 Firestone Lane, Northampton, MA 01060, USA, Email larry.bernstein@gmail.com

Received: October 18, 2017 | Published: December 01, 2017
Abbreviations: DM, diabetes mellitus; ER, endoplasmic reticulum; PD, protein degradation; PS, protein synthesis; PTM, posttranslational modification; ERAD, er-associated degradation; FFAs, free fatty acids; IAPP, islet amyloid polypeptide; PC, prohormone convertase

\section{Introduction}

The clinical feature of diabetes mellitus is unregulated hyperglycemia and impaired pancreatic insulin secretion. Diabetes is a systemic disease involving the pancreatic islet cell that produces insulin, and also the kidney, and eventual microvascular disease that has an impact on the heart and the brain. Opie ${ }^{1}$ described hyalinization of the pancreatic islet cells prior to the discovery of insulin, and Kimmelstein and Wilson ${ }^{2}$ observed hyalinization of the kidney glomeruli, also a hallmark of diabetic nephropathy, to which afferent arteriolar thickening (arteriolar nephrosclerosis) was later added. It can also be said that the hyalinization of the pancreatic beta cell and the kidneys is in either case amyloid deposit.

In recent years there has been considerable research that provides significant new insights into this common disease. Diabetes is now considered to be a disease of protein homeostasis, or proteostasis. ${ }^{3-5} \mathrm{It}$ has much in common with a number of neurodegenerative diseases, such as Alzheimer's disease, and a good number of others. In all of these diseases there is the accumulation of amyloid or another misfolded protein that is toxic to the cell involved.

\section{The structural impairment in diabetes mellitus}

It has been proposed that the main problem may not be in a specific amino acid sequence in the primary structure of the protein. A structural misfolding abnormality results in damage to the cell, and particularly creates a distortion of the cell membrane. This has been referred to a 'molecular ageing' which corresponds to nonenzymatic modifications causing progressive irreversible damage to proteins. ${ }^{3}$ Proteostasis is the cellular molecular process that is protein homeostasis and it is necessary for the function and integrity of the cell. This is the system responsible for maintenance of degradation systems (the proteasome and lysosomes), folding systems (dependent on chaperones), and enzymatic mechanisms of protein repair. ${ }^{3}$ There are also nonenzymatic post-translational modifications that occur as a result of hyperglycemia or increased production of reactive oxygen species in the development of diabetes mellitus (DM). A progressive loss of the ability of cells to degrade or repair altered proteins occurs as the impaired proteins accumulate. This is related to the impaired function of the endoplasmic reticulum (ER) under stress that leads to the accumulation. This leads to the b-cell damage in the pancreatic islet cell. The accumulation of damaged proteins results in a dysregulation of cell behavior in the pathogenesis of DM.

\section{Ubiquitin-proteasome pathway of protein degradation}

The ubiquitin-dependent proteasome pathway ${ }^{4}$ is the system by which a large proportion of abnormal or damaged intracellular proteins (about $80 \%$ ) are degraded and removed. Impairment of $26 \mathrm{~S}$ proteasome functioning results in non-enzymatic modifications altered by limited ubiquitinylation as a result of inaccessible target lysine residues. Consequently, damaged proteins accumulate and limit their degradation by inhibiting both autophagosome formation and lysosomal enzymes.

Insulin signaling regulates protein synthesis and degradation, posttranslational modifications in tissues, and coordinates proteostasis. ${ }^{4}$ It is important for amino acid flux, for the regulation of protein quality control, and for turnover of mitochondrial protein. Insulin deficiency in T1DM is related to a catabolic state with profound muscle wasting and nitrogen excretion because of the severe insulin insufficiency so that the hyperglycemia is coupled with inability of glucose to enter the cell. As a result, this is accompanied by the breakdown of lean body mass. The catabolic state of insulin deficiency results in mitochondrial function impairment that is associated with increased reactive oxygen species production. This leads to oxidative damage of proteins and impairs the regulation of proteostasis. The rate of protein turnover in tissues is variably influenced by insulin.

Muscle protein degradation (PD) is greatly increased in the insulin-deficient state. ${ }^{5}$ Amino acids are transported from muscle to the splanchnic bed, where both protein synthesis (PS) and PD are increased. In the fed state, muscle mass is 
maintained mainly under the influence of insulin and amino acids. Decreased insulin signaling occurs with fasting or in diabetes. Consequently, increased protein degradation occurs as a result of increased translocation and transcription of critical mediators of ubiquitin-proteasome and autophagy lysosome systems. ${ }^{4}$ Associated with this observation, diabetic patients have significantly greater oxidative protein damage compared with nondiabetic individuals. In addition, Apo A-1 undergoes accelerated oxidative posttranslational modification (PTM) in individuals with T1DM who are deprived of insulin.

\section{ER stress response}

In order to adapt to endoplasmic reticulum (ER) stress conditions, the cells respond in three distinct ways: transcriptional induction of ER chaperones, translational attenuation, and ER-associated degradation (ERAD). ${ }^{5}$ When ER functions are severely impaired, the cell is eliminated by apoptosis. The b-cell is a susceptible cell for ER stress and ER stress mediated apoptosis. ER dysfunction causes proteotoxicity in the ER, collectively termed "ER stress". ${ }^{5}$ ER stress activates ER stress transducer proteins such as Ire 1, PERK and ATF6. These signals are transmitted and lead to induction of CHOP. Ire 1 induces JNK activation. Caspase 12 is activated in response to ER stress. When b-cell overload induces chronic ER stress, it causes b-cell mass via apoptosis. This is referred to as "pancreatic b-cell exhaustion".

\section{Cell death pathway}

Two important pathways in the ER are apoptosis and autophagy, which are considered to play important roles in insulin insufficiency in $\mathrm{T} 1$ and T2DM. In addition, the ER acts as a monitor of proteostasis in insulin secretion. In this process, pro- and antiapoptotic $\mathrm{Bcl}-2$ proteins control cytochrome $\mathrm{c}$ release and cytochrome $\mathrm{c}$ binds to APAF-1 in mitochondria, which becomes activated procaspase -9 , and this is concurrent with the formation of procaspase-8. FADD is formed and activates procaspase -8 , followed by the activation of caspase $-3 .{ }^{6}$ This completes the death receptor pathway, termed programmed cell death. It is the mechanism by which the cell degrades malfunctioning proteins, organelles and soluble molecules by integration of lysosomes and double-membraned autophagosomes, which fuse with lysosomes for hydrolysis or degradation of misfolded proteins or membranes through the process of autophagy.

Hyperglycemia, increased free fatty acids (FFAs), islet amyloid polypeptide (IAPP), chronic low-grade ongoing inflammation and oxidative stress can induce protein misfolding in DM. ${ }^{6}$ ER stress induces misfolding and eventually $\beta$-cell death occurs via apoptosis. Activation of mTORC1 inhibits autophagy. Mammalian target of rapamycin 1 (mTORC1) decreases lysosomal degradation and its inhibition stimulates autophagy and prevents ERstress activated $\beta$-cell apoptosis.

SQSTM1/p62 deficient mice had severe hyperglycemia and consequently diabetes. Consequently, SQSTM1/p62 might be diminished secondary to increased autophagy via mTORC1 inhibition, and mTORC1 inhibitors could not stimulate LC3-II expression in beta cells because LC3-II accumulation was prevented by increased autophagosome formation.

\section{ER stress and fatty acid induced apoptosis}

The pro-apoptotic fatty acid palmitate triggers a comprehensive
ER stress response in MIN6 cells, a model of beta cell dysfunction that is not seen using non-apoptotic fatty acid oleate. This was accompanied by upregulated marker genes of ER stress in the islets of $\mathrm{db} / \mathrm{db}$ mice. This was evidenced by the heat shock protein A5 (HSPA5) in MIN6 cells that was overproduced and protected against lipid induced apoptosis. The existence of ER stress was confirmed by increased processing (activation) of $\mathrm{X}$-box binding protein 1 (Xbp1) mRNA. Of additional interest was the finding of increased islet protein production of HSPA5, DDIT3, DNAJC3 and BCL2-associated X protein in pancreas sections of type 2 diabetes subjects. The results of the study indicate that overproduction of the ER chaperone HSPA5 works against the effects of palmitate, that ER stress is required for beta cell lipoapoptosis and for developing type 2 diabetes. ${ }^{7}$ This suggests that the contribution of obesity and elevations in circulating fatty acids have an effect on the capacity of pancreatic beta cells to cope with this enhanced fatty acid supply. This study also linked ER stress selectively to lipoapoptosis. Nutritional excess results in a metabolically driven, low-grade, chronic inflammatory state, referred to as "metaflammation". ${ }^{8}$ This condition targets organs and tissues and it adversely affects systemic homeostasis. Endoplasmic reticulum dysfunction is another important feature of chronic metabolic disease that is also linked to both metabolic and immune regulation.

\section{ER stress and B-cell destruction}

ER stress requires nitric oxide generation, depletion of $\mathrm{ER} \mathrm{Ca}^{2+}$ stores and induction of DDIT3 to cause b-cell destruction. ER stress is a mechanism that integrates diverse aspects of type 2 diabetes into an understanding of its pathology. ${ }^{7}$ For instance, in Akita mice, a folding mutation in proinsulin is responsible; in Wolfram syndrome $\mathrm{ER} \mathrm{Ca}^{2+}$ handling is compromised; and Walcott-Rallison syndrome involves a defective gene encoding EIF2AK3. Thus, ER stress appears sufficient to cause beta cell apoptosis. Thus HSPA5 and DNAJC3 are secondary to ER stress, support the presence of ER stress, and suggest a mechanism for the apoptosis related to the reductions in beta cell mass that contribute to type 2 diabetes in humans.

The Wolcott-Rallison syndrome of infantile diabetes and mutations that prevent the a-subunit of eIF2 from being phosphorylated, block b-cell development, and impair gluconeogenesis are related to PERK. ${ }^{9}$ Synthetic function of islets of Langerhans from PERK-/- mice supports a model whereby ER stress is linked to the secretory activity of the b-cell. The level of ER stress in the b-cells would be increased in the hypersecretory state of insulin resistance. Thus, prolonged ER stress would be expected to be related to the degradation of $b$ cell function that precedes metabolic decomposition in the insulinresistant patient.

\section{Amylin peptide and amyloid generation}

Amylin is primarily responsible for classifying type II diabetes as an amyloid (protein misfolding) disease with the potential to aggregate into toxic nanoparticles. Amylin is derived from cleavage of preProIAPP at the N-terminal end yielding Pro-IAPP, which is post-translationally processed by the prohormone convertase (PC2). With respect to amylin peptide, the 22-27 region coils into amyloid fibrils, the 11-20 region binds to amylin with a high affinity, and the 14-18 region is the recognition site for amylin binding. ${ }^{10}$ ROS is damaging to DNA and oxidizes the constituent amino acids of proteins and polyunsaturated fatty acids. Consequently, oxidation by ROS causes detrimental changes in membrane fluidity, permeability, and metabolic functions. ${ }^{10}$ 
Protein aggregation is a generic property of polypeptide chains linked to their peptide backbone that may not depend on specific amino acid sequences. ${ }^{11}$ It has been suggested that the toxic effects of protein aggregates, mainly in their pre-fibrillar organization, result from common structural features, unrelated to specific sequences of side chains. In protein evolution, the negative selection against molecules with significant tendency to aggregate is related to the development of the complex molecular machineries that hinder the appearance of misfolded proteins and their toxic aggregates.

\section{Protein quality control and aggregation}

Protein evolution has a previously unappreciated constraint. A functional sequence endowed with a tendency to aggregate when it performs its biological function would have been discarded in order to provide cells with functional and stable proteins. This would ensure the quality control of protein folding. These mechanisms involve molecular chaperones both in the cytosol (heat-shock proteins, crystallins, prefoldin, Hsc70,) and in the endoplasmic reticulum (Bip, Grp94, calnexin) and the ubiquitin-proteasome pathway. ${ }^{11}$ These mechanisms ensure folding of polypeptide chains, and avoid inappropriate interactions of polypeptides to misfold. As a result of this quality control, a significant percentage of the proteins maturating in the ER are cleared before they can fold. In addition to amyloidoses, other protein misfolding diseases that deposit protein aggregates are known. Serine protease inhibitors such as a1-antitrypsin, antithrombin and plasminogen activator inhibitor 1 may be destabilized by specific mutations. For example, 1 out of over 70 a1-antitrypsin mutants aggregate into the endoplasmic reticulum of hepatocytes leading to liver disease in a1-antitrypsin deficiency.

\section{Acknowledgements}

None.

\section{Conflict of interest}

The author declares no conflict of interest.

\section{References}

1 Clark A, Moffitt J. Pancreatic Islet Amyloid and Diabetes. In: Protein Reviews Uversky VN, Fink AL, editors. Protein Misfolding, Aggregation, and Conformational Diseases. USA: Springer; 2007. p. 199-216.

2 Runyan JW, Hurwitz D, Robbins SL. Effect of Kimmelstiel-Wilson Syndrome on Insulin Requirements in Diabetes. $N$ Engl $\mathrm{J} \mathrm{Med}$. 1955;252(10):388-391.

3 Jaisson S, Gillery P. Impaired proteostasis: role in the pathogenesis of diabetes mellitus. Diabetologia. 2014;57(8):1517-1527.

4 James HA, O’Neill BT, Nair KS. Insulin Regulation of Proteostasis and Clinical Implications. Cell Metab. 2017;26(2):310-323.

5 Araki E, Oyadomari S, Mori M. Endoplasmic Reticulum Stress and Diabetes Mellitus. Intern Med. 2003;42(1):7-14.

6 Demirtas L, Guclu A, Erdur FM, et al. Apoptosis, autophagy \& endoplasmic reticulum stress in diabetes mellitus. Indian J Med Res. 2016;144(4):515-524

7 Laybutt DR, Preston AM, Åkerfeldt MC, et al. Endoplasmic reticulum stress contributes to beta cell apoptosis in type 2 diabetes. Diabetologia. 2007;50(4):752-763.

8 Hummasti S, Hotamisligil GS. Endoplasmic reticulum stress and inflammation in obesity and diabetes. Circ Res. 2010;107(5):579-591.

9 Harding HP, Ron D. Endoplasmic Reticulum Stress and the Development of diabetes: a review. Diabetes. 2002;51(Suppl 3):S455-S461.

10 Pillay K, Govender P. Amylin Uncovered: A Review on the Polypeptide Responsible for Type II Diabetes. BioMed Res Internl. 2013;2013:17.

11 Stefani M. Protein misfolding and aggregation: new examples in medicine and biology of the dark side of the protein world. Biochim Biophys Acta. 2004;1739(1):5-25. 